\title{
High Blood Viscosity Is Closely Associated With Cigarette Smoking and Markedly Reduced by Smoking Cessation
}

Sayaka Shimada; Koji Hasegawa, MD, PhD; Hiromichi Wada, MD, PhD; Sachiko Terashima; Noriko Satoh-Asahara, MD, PhD; Hajime Yamakage; Shuji Kitaoka, MD; Masaharu Akao, MD; Akira Shimatsu, MD, PhD; Yuko Takahashi, MD, PhD

\begin{abstract}
Background: Cigarette smoking is an independent risk factor for cardiovascular events such as myocardial infarction and stroke. To date, a useful and convenient method of predicting such events in smokers has not been established. The rheological properties of blood assessed by the microchannel method reflect the blood's viscosity and the state of microthrombus formation, which may predict cardiovascular thrombotic events.
\end{abstract}

Methods and Results: Blood fluidity was assessed in 74 smoking patients (54 men, 20 women, mean age 57.9 years) by measuring the blood passage time (BPT) in an aliquot (100 $\mu$ l) of blood using the Micro Channel Array Flow Analyzer. BPT was significantly related with smoking variables such as daily consumption of tobacco $(r=0.236, P=0.044)$, Brinkman's index $(r=0.252, P=0.033)$, the Fagerstrom Test for Nicotine Dependence $(r=0.257$, $\mathrm{P}=0.029)$, and the score of a self-rating depression scale $(r=0.236, P<0.05)$. Multivariate regression analysis revealed that an independent BPT determinant was daily consumption of tobacco $(r=0.326, P=0.045)$. Furthermore, smoking cessation markedly decreased BPT from 63.0 s to $49.7 \mathrm{~s}(\mathrm{P}=0.002)$ at 3 months after the start of therapy.

Conclusions: Unfavorable blood rheology is closely associated with cigarette smoking and may reflect increased cardiovascular risk in smokers. The study results also suggest that such risk can be reduced after only 3 months of smoking cessation. (Circ $J$ 2011; 75: 185-189)

Key Words: Blood rheology; Cardiovascular risk; Smoking

B lood rheology or fluidity, which is expressed as "viscous" or "non-viscous", reflects blood viscosity and the state of blood cells. Fluidity has been reported to be reduced (ie, viscosity is increased) in patients with lifestyle-related diseases such as hypertension. ${ }^{1}$ Furthermore, reduced blood fluidity has been suggested to lead to cardiovascular thrombotic events such as myocardial and cerebral infarction. ${ }^{2}$ The Micro Channel Array Flow Analyzer (MCFAN) is an instrument that measures blood fluidity by a microchannel method using a capillary model. ${ }^{3}$ This device analyzes the fluidity of blood passing a silicone-coated microchannel array according to its viscosity, microthrombus formation etc. The MC-FAN is superior to other methods in terms of accuracy and reproducibility, and evidence that it is markedly useful for the evaluation of blood fluidity and microcirculatory systems has accumulated. ${ }^{4-6}$
Smoking is a major risk factor for cardiovascular diseases, associated with endothelial dysfunction, ${ }^{7}$ but a decrease in blood fluidity in smokers or the effect of smoking cessation on viscosity has not yet been evaluated. Therefore, we measured blood fluidity in smokers, analyzed its correlation with various factors related to smoking, and examined whether quitting smoking improves fluidity.

\section{Methods}

\section{Subjects}

Blood fluidity and various parameters were analyzed in 74 consecutive patients who visited the Smoking Cessation Clinic, National Hospital Organization, Kyoto Medical Center between August 2008 and June 2009 and consented to this study. Those who were treated with an antiplatelet or anti-

Received April 6, 2010; revised manuscript received July 29, 2010; accepted August 11, 2010; released online November 7, 2010 Time for primary review: 33 days

Clinical Research Institute (S.S., K.H., H.W., N.S.-A., H.Y., M.A., A.S.), Health Screening Center (S.T., S.K.), Kyoto Medical Center, National Hospital Organization, Kyoto; and Health Administration Center, Nara Women's University, Nara (Y.T.), Japan

Mailing address: Koji Hasegawa, MD, PhD, Director, Division of Translational Research, Clinical Research Institute, Kyoto Medical Center, National Hospital Organization, 1-1 Mukaihata-cho, Fukakusa, Fushimi-ku, Kyoto 612-8555, Japan. E-mail: koj@kuhp. kyoto-u.ac.jp

ISSN-1346-9843 doi:10.1253/circj.CJ-10-0335

All rights are reserved to the Japanese Circulation Society. For permissions, please e-mail: cj@j-circ.or.jp 
A.

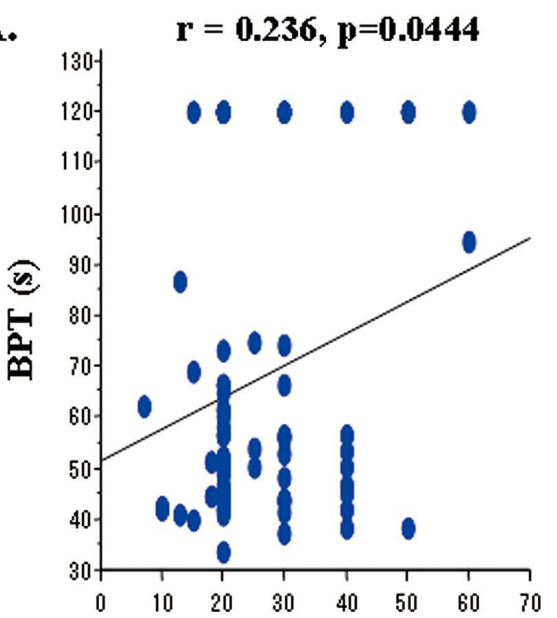

Daily consumption of tobacco (n)
C.

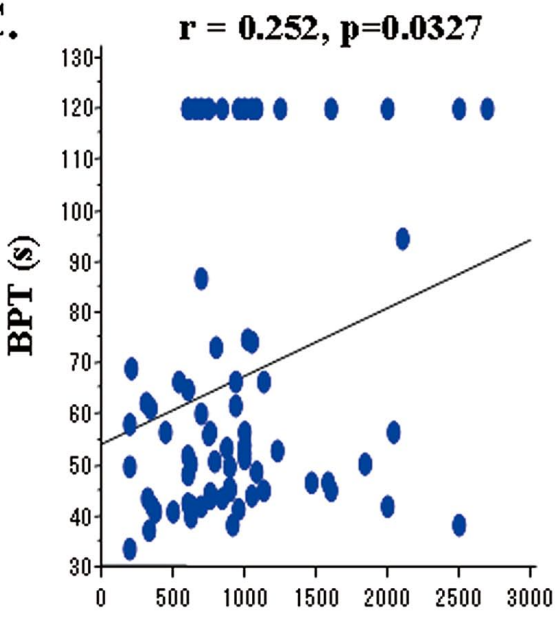

Brinkman's Index
B.

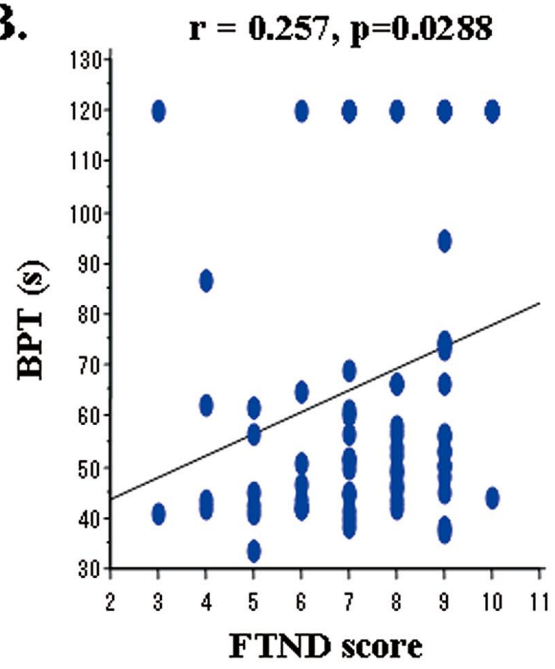

D.

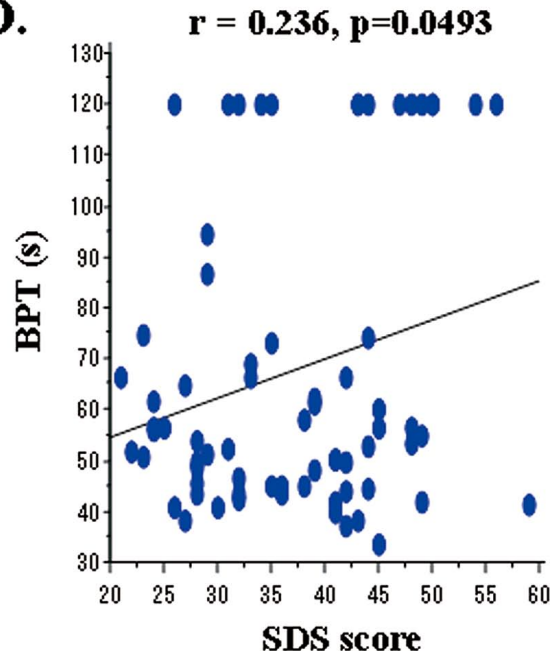

Figure 1. Scatter grams of correlations between the number of cigarettes smoked per day (A), Fagerstrom Test for Nicotine Dependence (FTND) (B), Brinkman index (C), or self-rating depression score (D) and the blood passage time (BPT).

coagulant were excluded. Among 74 patients, 13 had received antihypertensive agents, 3 received statins, and 10 antidiabetic drugs.

Blood fluidity was evaluated before and after quitting smoking in 17 patients who repeatedly visited the Smoking Cessation Clinic, National Hospital Organization, Kyoto Medical Center for more than 3 months during the study period and who successfully quit smoking.

This study was performed after explaining the objective to the subjects in writing and obtaining their consent. It was performed with the approval of the Ethical Review Board, National Hospital Organization, Kyoto Medical Center.

\section{Evaluation of Depression}

The severity of depression was evaluated using a questionnaire based on the Self-rating Depression Scale (SDS). The questionnaire was answered by the patients, the answers were checked by the study staff, and the patients were asked to fill it in again if there were items that they had omitted or if mistakes had been made on answering. ${ }^{8,9}$

\section{Central Blood Pressure}

The aortic pressure waves were estimated from pulse waves of the peripheral radial artery measured with an Omron HBM-9000AI and the augmentation index (AI) was determined as previously described. ${ }^{10}$

\section{Blood Sampling and Evaluation of the Blood Fluidity}

Blood was obtained from the patient's antecubital vein $2-3 \mathrm{~h}$ after lunch to determine hemoglobin $\mathrm{A}_{1 \mathrm{c}}\left(\mathrm{HbA}_{1 \mathrm{c}}\right)$, low-density lipoprotein-cholesterol (LDL-C), and high-density lipoprotein-cholesterol (HDL-C) levels. The blood passage time (BPT) was measured with the MC-FAN HR300 rheometer (MC Lab.), as reported previously. ${ }^{6,11}$ First, the microchannel passage time of $100 \mu \mathrm{l}$ of physiologic saline was measured as a control, and then that of venous blood obtained from the patient with 5\% heparinization was determined. The BPT of the patient was expressed after correction for the passage time of physiologic saline. A BPT exceeding $120 \mathrm{~s}$ was expressed as $120 \mathrm{~s}$. 


\begin{tabular}{|c|c|c|c|c|}
\hline & \multicolumn{2}{|c|}{ Univariate } & \multicolumn{2}{|c|}{ Multivariate } \\
\hline & $r$ value & $P$ value & $r$ value & $P$ value \\
\hline Age, years & 0.112 & 0.3461 & - & - \\
\hline Body weight, $\mathrm{kg}$ & 0.090 & 0.4672 & - & - \\
\hline $\mathrm{SBP}, \mathrm{mmHg}$ & 0.060 & 0.6180 & - & - \\
\hline $\mathrm{DBP}, \mathrm{mmHg}$ & 0.198 & 0.0953 & - & - \\
\hline $\mathrm{HbA}_{1 \mathrm{c}}, \%$ & 0.045 & 0.7186 & - & - \\
\hline $\mathrm{LDL}-\mathrm{C}, \mathrm{mg} / \mathrm{dl}$ & 0.049 & 0.6979 & - & - \\
\hline $\mathrm{HDL}-\mathrm{C}, \mathrm{mg} / \mathrm{dl}$ & -0.078 & 0.5335 & - & - \\
\hline Duration of smoking, years & 0.130 & 0.2722 & - & - \\
\hline Age at start of smoking, years & -0.098 & 0.4110 & - & - \\
\hline Daily consumption of tobacco, $\mathrm{n}$ & 0.236 & 0.0444 & 0.326 & 0.0446 \\
\hline FTND score & 0.257 & 0.0288 & - & - \\
\hline Brinkman's index & 0.252 & 0.0327 & - & - \\
\hline TDS score & 0.072 & 0.5471 & - & - \\
\hline Confidence in smoking cessation, \% & -0.111 & 0.3914 & - & - \\
\hline $\mathrm{Al}, \%$ & -0.050 & 0.7246 & - & - \\
\hline SDS score & 0.236 & 0.0493 & - & - \\
\hline
\end{tabular}

SBP, systolic blood pressure; DBP, diastolic blood pressure; $\mathrm{HbA}_{1 c}$, hemoglobin $\mathrm{A}_{1 c}$; $\mathrm{LDL}-\mathrm{C}$, low-density lipoproteincholesterol; HDL-C, high-density lipoprotein-chlesterol; FTND, Fagerstrom Test for Nicotine Dependence; TDS, Tobacco Dependence Screener; AI, augmentation index; SDS, Self-rating Depression Scale.

\section{Smoking Cessation Clinic}

During the initial consultation, nicotine dependence was assessed with both the Fagerstrom Test for Nicotine Dependence $(\text { FTND })^{12}$ and the Tobacco Dependence Screener (TDS). ${ }^{13}$ In both tests, scores range from 0 to 10 , with higher score indicating more severe nicotine dependence. The number of cigarettes smoked per day was determined by asking the smoker, "On average, in the past month, how many cigarettes did you smoke per day?". Smokers were asked to rate their confidence in their ability to abstain from smoking cigarettes in the next 3 months on a scale of $0-100 \%$.

Anti-smoking treatment was conducted according to the Standard Procedures for Anti-Smoking Treatment (issued in March 2006 by the Japanese Circulation Society, Japan Lung Cancer Society, and Japanese Cancer Association). The patients were examined on their first visit and 2, 4, 8, and 12 weeks thereafter and treated with nicotine patches or oral varenicline. On their repeat visits, whether or not the patients had maintained smoking cessation was checked, and specific advice concerning the continuation of cessation was given. At the end of the anti-smoking treatment (after 12 weeks), whether or not cessation had been maintained was evaluated. A patient was judged to have succeeded in quitting smoking with an expiratory carbon monoxide concentration of $7 \mathrm{ppm}$ or less and the patient's affirmation of not smoking. The attempt to quit smoking was judged to have been unsuccessful when the patient stopped visiting during the treatment period or continued visiting but failed to quit smoking.

\section{Statistical Analysis}

Data are presented as the mean $\pm \mathrm{SE}$, and $\mathrm{P}<0.05$ was considered statistically significant. Statistical analyses were performed as previously described. ${ }^{14}$ The correlations of blood fluidity with various parameters were examined according to Pearson's correlation coefficient. Factors that determine blood fluidity were analyzed using multiple logistic regression analysis. The blood fluidity values before and after quitting smoking were compared by the Mann-Whitney U-test. $\mathrm{P}<0.05$ was regarded as significant.

\section{Results}

\section{Correlations of BPT With Various Parameters in Patients Treated at the Smoking Cessation Clinic}

The 74 subjects in this study who visited our Smoking Cessation Clinic consisted of 54 males and 20 females, with a mean age of $57.9 \pm 1.6$ years. The mean male age was $61.9 \pm$ 1.6 years, and the mean female age was $47.4 \pm 3.0$ years. The duration of smoking at the initial examination was $37.5 \pm$ 1.5 years, age at the beginning of regular smoking was $19.3 \pm$ 0.5 years, number of cigarettes smoked per day was $26.0 \pm 1.3$, FTND score was 7.3 \pm 0.2 , Brinkman index was 953.8 \pm 65.5 , TDS score was $8.4 \pm 0.2$, degree of self-confidence to quit smoking was $42.3 \pm 3.8 \%$, AI was $77.6 \pm 2.3 \%$, and SDS score was $37.0 \pm 1.1$.

The BPT showed significant positive correlations with the number of cigarettes smoked per day (Figure 1A), FTND score (Figure 1B), and Brinkman index (Figure 1C). It was also significantly correlated with the SDS test score (Figure 1D), an index of depressive mood. However, the BPT showed no significant correlation with metabolic parameters such as body weight, systolic and diastolic blood pressures, $\mathrm{HbA}_{1 \mathrm{c}}$, LDL-C or HDL-C. There was no significant difference in the BPT between patients treated with $(n=13,70.9 \pm 8.2 \mathrm{~s})$ and without $(n=61,66.5 \pm 3.9 \mathrm{~s})$ antihypertensive agents, between patients treated with $(n=3,75.9 \pm 22.1 \mathrm{~s})$ and without $(n=71$, $66.9 \pm 3.5 \mathrm{~s})$ statins, or between patients taking $(\mathrm{n}=10,68.1 \pm$ $9.2 \mathrm{~s})$ and those not taking $(\mathrm{n}=64,67.1 \pm 3.8 \mathrm{~s})$ antidiabetic agents. The SDS test score was significantly correlated with the FNTD score $(\mathrm{r}=0.658, \mathrm{P}<0.001)$, TDS score $(\mathrm{r}=0.696$, $\mathrm{P}<0.001)$ daily consumption of tobacco $(\mathrm{r}=0.528, \mathrm{P}<0.001)$, duration of smoking $(\mathrm{r}=0.378, \mathrm{P}<0.001)$, and Brinkman's index $(r=0.375, \mathrm{P}<0.001)$. On multivariate analysis using the BPT as the dependent variable, the number of cigarettes smoked per day was found to be an independent related factor $(\mathrm{r}=0.326, \mathrm{P}=0.045)$ (Table 1$)$. 
A.

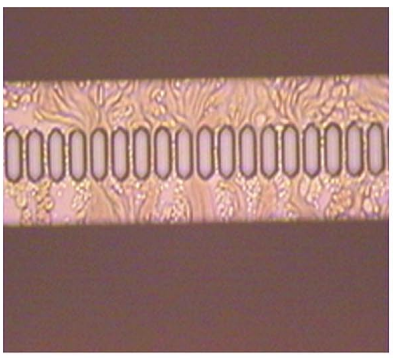

before

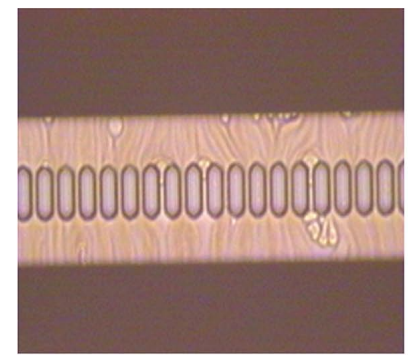

after

B.

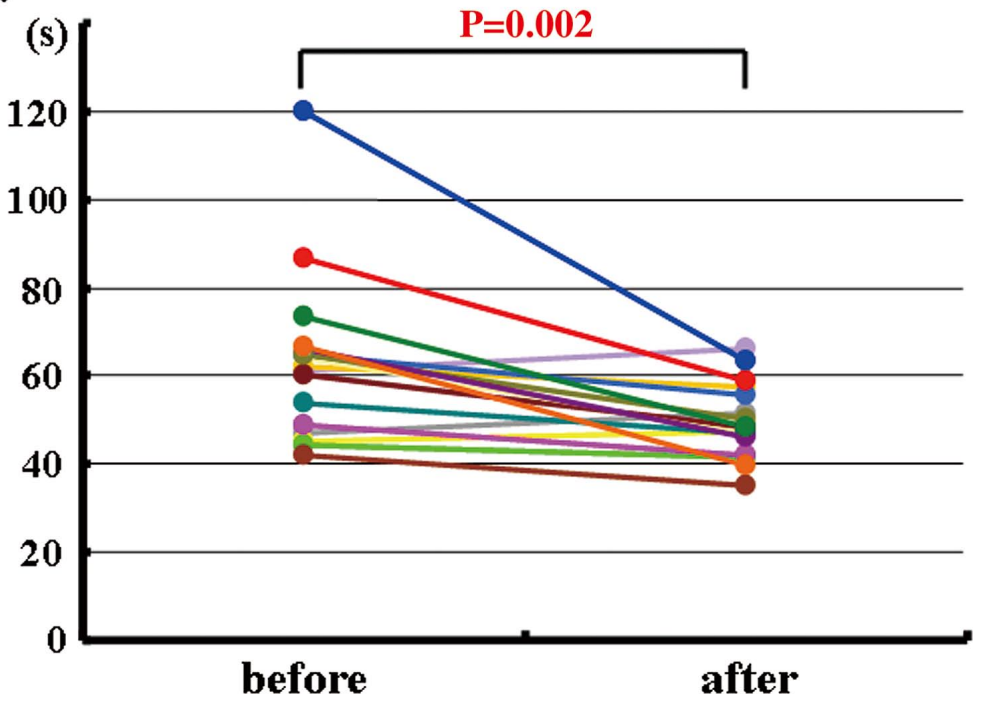

Figure 2. Blood passage time measured by the Micro Channel Array Flow Analyzer (MC-FAN) before and after smoking cessation. (A) Representative photographs of blood passing through microchannels before and after smoking cessation. Before, at the time of initial consultation at the smoking cessation clinic; After, at 3 months after the initial consultation. (B) Blood passage time measured by the MC-FAN before and after smoking cessation.

\section{Changes in the BPT in Patients Who Successfully Quit Smoking}

The 17 patients who successfully quit smoking on outpatient treatment in this study consisted of 16 males and 1 female, with a mean age of $61.5 \pm 2.7$ years. The initial prescription was nicotine patches (Nicotinell TTS) in 6 and varenicline (Champix) in 11, but the nicotine patch therapy was changed to varenicline during the course of treatment in 1 patient. Body weight significantly $(\mathrm{P}<0.05)$ increased from $69.5 \pm$ $3.1 \mathrm{~kg}$ at the beginning to $70.9 \pm 3.1 \mathrm{~kg}$ at the end. The BPT significantly shortened from the initial examination to the end of the 3-month period of insured treatment $(63.0 \pm 4.5$ to $49.7 \pm$ $2.0 \mathrm{~s}, \mathrm{P}=0.002$ ) (Figure 2). The improvement was marked in patients with an initial BPT of $50 \mathrm{~s}$ or longer $(\mathrm{n}=12,70.3 \pm 5.1$ to $52.3 \pm 3.5 \mathrm{~s}, \mathrm{P}=0.002$ ), but was unremarkable and non-significant in those with an initial BPT shorter than $50 \mathrm{~s}(\mathrm{n}=5$, $45.5 \pm 1.2$ to $43.5 \pm 5.7 \mathrm{~s}, \mathrm{P}=0.450$ ). The BPT did not improve in patients who were unsuccessful in ceasing smoking $(n=3$, $77.3 \pm 22.9$ to $91.7 \pm 28.3 \mathrm{~s})$.

Changes in the BPT were compared according to the treatment. A patient who could not quit smoking on nicotine patch treatment but successfully quit on varenicline administration was included in the varenicline group. At the initial examination, the BPT tended to be longer in the varenicline group $(n=11,68.6 \pm 6.1 \mathrm{~s})$ than in the nicotine patch group $(\mathrm{n}=$ $6,52.8 \pm 4.1 \mathrm{~s})(\mathrm{P}=0.097)$. In the varenicline group, the BPT decreased significantly from before to after quitting smoking (68.6 \pm 6.1 to $52.0 \pm 2.7 \mathrm{~s}, \mathrm{P}=0.007$ ). In the nicotine patch group, it also decreased after quitting smoking, although not significantly (52.8 \pm 4.1 to $45.4 \pm 2.0 \mathrm{~s}, \mathrm{P}=0.182)$.

\section{Discussion}

Reduced blood fluidity has been suggested to lead to thrombotic events, such as cerebral and myocardial infarction, which are the most serious complications of lifestyle-related diseases. ${ }^{1,2}$ Analysis using the MC-FAN has revealed that blood viscosity increases when cardiovascular risk factors such as hypertension, dyslipidemia, and obesity are present. $^{3-5}$ We have also reported that blood viscosity increases markedly in patients with metabolic syndrome and decreases with weight reduction. ${ }^{6}$ However, there has been no detailed analysis using the MC-FAN of reduced blood fluidity (ie, increased blood viscosity) in smokers. In this study, the BPT was significantly correlated with the number of cigarettes smoked per day, FTND score, and Brinkman index in patients who initially visited the Smoking Cessation Clinic. Also, multivariate analysis showed that the number of cigarettes smoked per day was a determinant of the BPT. From these observations, smoking is considered to be clearly related to an increase in blood viscosity.

Women's blood viscosity may alter before and after menopause. Unfortunately, we do not have information on the menstrual status of the female patients in the present study. Furthermore, the number of women $(n=20)$ in our study population did not appear to be sufficient to analyze differences 
in blood viscosity based on menstrual status. There was no significant difference in the BPT between male $(n=54,70.2 \pm$ $4.2 \mathrm{~s})$ and female $(\mathrm{n}=20,59.2 \pm 6.1 \mathrm{~s})$ patients, or between female patients above $(n=10,53.4 \pm 7.7 \mathrm{~s})$ and below $(n=10$, $65.1 \pm 9.6 \mathrm{~s}$ ) the age of 50 , at which menopause occurs on average. However, further studies are needed on the affect of sex and menstrual status on blood viscosity. Nevertheless, analysis of the total population including both sexes clearly indicated a close association between smoking-associated variables and the BPT. Thus, the suggestion of a close association between smoking and increased blood viscosity may be valid.

In our clinic, when the score of the SDS test is in the range of major depression $(>60)$, we always consult psychiatrists before starting smoking cessation therapy. However, the SDS test score of smoking patients in the present study ranged from 21 to 59, and no patient showed a score above 60 . These findings suggest that the patients in this study were not in the "diseased state of major depression", but in a "latent depressive state". We previously reported that the incidence of latent depression was high in smokers even without a clear history of psychiatric disorders. ${ }^{8}$ Interestingly, an increase in blood viscosity was also significantly correlated with the SDS test score, which is an index of depression. Details of the mechanism of the relationship of depression with a low blood fluidity are unknown. Patients with depression or latent depression show a marked dependence on smoking and have difficulty in quitting. ${ }^{9}$ The present study demonstrates that the SDS test score was significantly correlated with nicotine dependency and number of cigarettes smoked by patients. The correlations of the SDS score with smoking variables suggest that a latent depressive state significantly contributes to a high blood viscosity (reduced blood fluidity) caused by cigarette smoking. However, these data do not rule-out the possibility that a latent depressive state is directly involved in reduced blood fluidity. Type A characteristics are a wellknown risk factor for cardiovascular events such as myocardial infarction and stroke. Further evaluation is necessary to clarify whether or not a latent depressive state is directly associated with reduced blood fluidity.

The BPT was significantly shortened in patients who successfully quit smoking after 3 months of anti-smoking treatment. The shortening was more marked in patients who showed a higher blood viscosity at the initial examination. An early improvement in blood fluidity after the onset of anti-smoking treatment may suggest an early decrease in cardiovascular risk. The BPT was shortened in patients treated with varenicline and those treated with nicotine patches. However, shortening was marked and significant in the former group but was not significant in the latter. Varenicline can be used more safely than nicotine patches in patients with cerebral or myocardial infarction. At our hospital, varenicline is used as the first choice for smoking cessation therapy in patients with a high cardiovascular risk, such as those with a history of cerebral or myocardial infarction. In addition, when smoking patients exhibit relatively higher amounts of tobacco consumption, we preferentially choose varenicline rather than a nicotine patch. In fact, the number of cigarettes smoked per day tended to be larger in the present varenicline group $(25.0 \pm 11.3)$ than in the nicotine patch group $(19.7 \pm$ 0.8 ). The BPT tended to be longer in the varenicline group than in the nicotine patch group at the initial examination. A relatively high consumption of tobacco in the varenicline group may have contributed to a longer BPT in this group at baseline. The shortening of the BPT after quitting smoking is considered to have been essentially related to quitting smoking itself, but a direct effect of varenicline on blood fluidity cannot be excluded.

Blood fluidity was closely correlated with factors related to smoking and was improved by quitting. Evaluation of blood fluidity using the MC-FAN is not only highly reproducible but the device also enables visual observation of blood passing through the microchannels. Thus many of the smokers realized that their blood was viscous, which renewed their motivation to quit smoking. This study suggests the usefulness of blood fluidity as an index of cardiovascular event risk in smokers, an index of the effectiveness of anti-smoking treatment, and as a tool to encourage patients to quit smoking.

\section{Acknowledgments}

This study owes much to the generous cooperation of Messrs Yuko Iida, Shuichi Ura, Akira Yamada, Yosuke Sasaki, and Kazuya Muranaka at the National Hospital Organization Kyoto Medical Center. This study was partly sponsored by a Grant-in-Aid from the Ministry of Health, Labour and Welfare for Research on Economic Effects of Various Strategies for Smoking Cessation.

\section{References}

1. Lip GY, Beevers DG. Abnormalities of rheology and coagulation in hypertension. J Hum Hypertens 1994; 8: 693-702.

2. Tzoulaki I, Murray GD, Lee AJ, Rumley A, Lowe GD, Fowkes FG. Relative value of inflammatory, hemostatic, and rheological factors for incident myocardial infarction and stroke: The Edinburgh Artery Study. Circulation 2007; 115: 2119-2127.

3. Kikuchi Y, Sato K, Mizuguchi Y. Modified cellflow microchannels in a single-crystal silicon substrate and flow behavior of blood cells. Microvasc Res 1994; 47: 126-139.

4. Seki K, Sumino H, Nara M, Ishiyama N, Nishino M, Murakami M. Relationships between blood rheology and age, body mass index, blood cell count, fibrinogen, and lipids in healthy subjects. Clin Hemorheol Microcirc 2006; 34: 401-410.

5. Kotani K, Inata A, Araga S. Hemorheology by microchannel method in males with metabolic syndrome. Arch Med Res 2007; 38: $463-464$.

6. Satoh N, Kotani K, Wada H, Himeno A, Shimada S, Sasaki Y, et al. Unfavorable blood rheology is closely associated with arterial stiffness in obese patients. Endocr J 2009; 56: 915-918.

7. Yufu K, Takahashi N, Okada N, Shinohara T, Hara M, Saikawa T, et al. Influence of systolic blood pressure and cigarette smoking on endothelial function in young healthy people. Circ J 2009; 73: $174-178$.

8. Hasegawa K, Terashima S, Satoh N, Inoue M, Wada H, Itoh C, et al. Depressive state of patients on their initial visit to a smoking cessation clinic. Smoking Control Science 2008; 2: 23-26.

9. Wada H, Hasegawa K, Terashima S, Satoh N, Inoue M, Iida Y, et al. Self-rating depression scale score is a strong independent predictor of smoking cessation outcomes. Smoking Control Sci 2008; 2: 4-8.

10. Mahmud A, Feely J. Effects of passive smoking on blood pressure and aortic pressure waveform in healthy young adults: Influence of gender. Br J Clin Pharmacol 2003; 57: 37-43.

11. Seki K, Sumino H, Murakami M. Study on blood rheology measured by MC-FAN. Rinsho Byori 2003; 51: 770-775 (in Japanese).

12. Kawakami N, Takatsuka N, Inaba S, Shimizu H. Development of a screening questionnaire for tobacco/nicotine dependence according to ICD-10, DSM-III-R and DSM-IV. Addict Behav 1999; 24: $155-166$.

13. Thorndike AN, Regam S, McKool K, Pasternak RC, Swartz S, Torres-Finnerty N, et al. Depressive symptoms and smoking cession after hospitalization for cardiovascular disease. Arch Intern Med 2008; 168: 186-191.

14. Satoh N, Wada H, Ono K, Yamakage H, Yamada K, Nakano T, et al. Small dense LDL-cholesterol relative to LDL-cholesterol is a strong independent determinant of hypoadiponectinemia in metabolic syndrome. Circ J 2008; 72: 932-939. 